\title{
Extragonadal teratoma
}

INSERM

\section{Source}

INSERM. (1999). Orphanet: an online rare disease and orphan drug data base.

Extragonadal teratoma. ORPHA:883

Extragonadal teratoma is an extremely rare, benign or malignant germ cell tumor characterized, clinically, by a teratoma presenting in an extragonadal location (e.g. retroperitoneum, mediastinum, craniofacial or sacrococcygeal region, intraosseous, solid organs) and, histologically, by displaying well-differentiated structures, as well as immature elements. Presenting symptoms are variable depending on size and location of tumor. 